\title{
Update on the TMT laser guide star facility design
}

Corinne Boyer, Brent Ellerbroek, Mike Gedig, Edward Hileman, Richard Joyce, et al.

Corinne Boyer, Brent Ellerbroek, Mike Gedig, Edward Hileman, Richard Joyce, Ming Liang, "Update on the TMT laser guide star facility design," Proc. SPIE 7015, Adaptive Optics Systems, 70152N (10 July 2008); doi: 10.1117/12.787603

Event: SPIE Astronomical Telescopes + Instrumentation, 2008, Marseille, France 


\title{
Update on the TMT laser guide star facility design
}

\author{
Corinne Boyer**a ${ }^{*}, B_{\text {rent Ellerbroek }}^{\mathrm{a}}$, Mike Gedig ${ }^{\mathrm{b}}$, Edward Hileman $^{\mathrm{c}}$, Richard Joyce ${ }^{\mathrm{c}}$, Ming Liang $^{\mathrm{c}}$ \\ ${ }^{a}$ TMT Observatory Corp., 2632 E. Washington Blvd., Pasadena, CA 91107; \\ ${ }^{\mathrm{b}}$ Empire Dynamic Structures, 1515 Kingsway Ave., Port Coquitlam, B.C. Canada, V3C 1S2; \\ ${ }^{c}$ National Optical Astronomy Observatory, P.O. Box 26732, Tucson AZ 85726-6732
}

\begin{abstract}
The Thirty Meter Telescope (TMT) will implement a Laser Guide Star Facility (LGSF), which will generate up to nine $\mathrm{Na}$ laser beams in at least four distinct asterisms. The TMT LGSF conceptual design is based upon three 50W solid state, continuous wave, sum frequency $589 \mathrm{~nm}$ lasers and conventional beam transport optics. In this paper, we provide an update to the TMT LGSF conceptual design. The LGSF top end and the beam transfer optics have been significantly redesigned to compensate for the TMT telescope top end flexure, to adapt for the new TMT Ritchey-Chrétien optical design, to reduce the number of optical surfaces and to reduce the mass and volume. Finally, the laser service enclosure has been relocated within the telescope azimuth structure. This will permit the lasers to operate with a fixed gravity vector, but also requires further changes in the beam transport optical path.
\end{abstract}

Keywords: Thirty Meter Telescope, Adaptive Optics, Laser Guide Star, Beam Transfer Optics

\section{INTRODUCTION}

The initial Adaptive Optics (AO) architecture for the TMT has been defined to provide near-diffraction-limited wavefront quality and high sky coverage in the near infra-red (IR) for the early light TMT science instruments IRIS and IRMS $^{1,2,3}$. It is a Laser Guide Star (LGS) Multi Conjugate AO (MCAO) architecture consisting of (i) the Narrow Field IR AO System (NFIRAOS) ${ }^{4}$, which feeds up to 3 science instrument ports after sensing and correcting for wavefront aberrations introduced by the atmospheric turbulence and the telescope itself, and (ii) the Laser Guide Star Facility (LGSF) which generates multiple LGS in the mesospheric sodium layer with the brightness, beam quality and asterism geometry required by both NFIRAOS and later the $2^{\text {nd }}$ generation of TMT AO systems. The early light NFIRAOS will implement 2 Deformable Mirrors (DM) conjugated at 0km (61x61 DM) and 12km (73x73 DM) and 6 LGS Wavefront Sensors with 60x60 sub-apertures. It will operate at $800 \mathrm{~Hz}$.

A conceptual design of the LGSF was developed by NOAO and TMT in $2006^{5}$ to satisfy the LGSF requirements described in Table 1.

Table 1: LGSF Requirements.

\begin{tabular}{|c|c|}
\hline & Requirement \\
\hline Asterism & $\begin{array}{l}\text { - Generate the NFIRAOS asterism: } 1 \text { LGS on-axis, } 5 \text { LGS equally spaced on a circle of } \\
\text { radius of } 35 \text { arcsec } \\
\text { - Support additional asterisms for other AO modes with up to } 9 \text { LGS and radii varying } \\
\text { between } 5 \text { arcsec and } 510 \text { arcsec } \\
\text { - Switch rapidly from one asterism to another }\end{array}$ \\
\hline Lasers & $\begin{array}{l}\text { - Generate } 25 \mathrm{~W} \text { per LGS (17W minimum) using } 589.3 \mathrm{~nm} \text { solid state, Continuous Wave } \\
\text { (CW) or mode locked CW, Sum Frequency Generation (SFG) lasers ( } 150 \mathrm{~W} \text { total laser } \\
\text { power is required for NFIRAOS). } \\
\text { - This is anticipated to produce a signal level of } 900 \text { photon-detection-events per sub- } \\
\text { aperture at } 800 \mathrm{~Hz} \text {, assuming a sodium column density of } 4 \times 10^{13} \text {, a laser to sodium } \\
\text { coupling efficiency of } 130 \text { photons- } \mathrm{m}^{2} / \mathrm{s} / \mathrm{W} / \mathrm{ion}, \mathrm{TMT} \text { 's nominal parameter for end-to-end }\end{array}$ \\
\hline
\end{tabular}

*cboyer@tmt.org; phone 1626 395-1623; fax 1626 395-8909; www.tmt.org

Adaptive Optics Systems, edited by Norbert Hubin, Claire E. Max, Peter L. Wizinowich,

Proc. of SPIE Vol. 7015, 70152N, (2008)

0277-786X/08/\$18 $\cdot$ doi: $10.1117 / 12.787603$

Proc. of SPIE Vol. $701570152 \mathrm{~N}-1$ 


\begin{tabular}{|c|c|}
\hline & $\begin{array}{l}\text { optical throughput and atmospheric transmittance of } 0.19 \text {, a subaperture width of } 0.5 \mathrm{~m} \text {, and } \\
\text { a quantum efficiency of } 0.9 \text { for the LGS wavefront sensor detectors }{ }^{6} \text {. }\end{array}$ \\
\hline LGS Beam quality & Yield an output LGS with a far-field $1 / \mathrm{e}^{2}$ diameter no greater than $0.62 \operatorname{arcsec}$ \\
\hline LGS Tip Tilt Jitter & $\begin{array}{l}\text { - Maintain the asterism fixed on the sky as the telescope tracks the science field with a 1- } \\
\text { axis, 1-sigma tip/tilt jitter no greater than 50mas } \\
\text { - Implement necessary control and diagnostics systems: correction for slow (flexure) and } \\
\text { fast (windshake) laser beam perturbations }\end{array}$ \\
\hline LGS Polarization & Project $98 \%$ circularly polarized LGS beacons to maximize the photon return \\
\hline Beam Transfer Optics & Utilize conventional optics (not fibers) \\
\hline $\begin{array}{l}\text { Laser Launch } \\
\text { Telescope }\end{array}$ & $\begin{array}{l}\text { - Mount the Laser Launch Telescope behind the TMT secondary mirror to minimize the } \\
\text { elongation } \\
\text { - Laser Launch Telescope aperture: } 0.5 \mathrm{~m}\end{array}$ \\
\hline LGSF Throughput & $\begin{array}{l}\text { Yield an overall throughput of at least } 0.75 \text { from the output of the Lasers to the Laser } \\
\text { Launch Telescope }\end{array}$ \\
\hline LGSF Downtime & $\begin{array}{l}\text { - Operate with a downtime no greater than } 0.5 \%(0.26 \% \text { for the lasers }) \\
\text { - Implement multiple lasers and be operational with one laser down }\end{array}$ \\
\hline LGSF Safety & $\begin{array}{l}\text { Implement all necessary safety system to protect personnel, observatory systems, aircrafts, } \\
\text { satellites from laser light illumination and to protect neighboring telescopes from } \\
\text { projection of the LGS within their field of view. }\end{array}$ \\
\hline LGSF Upgrades & $\begin{array}{l}\text { Be upgradeable to provide LGS with signal level and beam quality required by an upgraded } \\
\text { version of the NFIRAOS system (120x120 order system). }\end{array}$ \\
\hline LGSF Budgets & $\begin{array}{l}\text { Must meet the TMT requirements in terms of mass, volume, power consumption, heat } \\
\text { dissipation, cooling, earthquake survival and accessibility. }\end{array}$ \\
\hline
\end{tabular}

The 2006 LGSF conceptual design was derived in part from the Gemini North and Gemini South telescopes LGSF design ${ }^{7}$. It consisted of three main sub-systems: (i) the Laser System, (ii) The Beam Transfer Optics (BTO) and Laser Launch Telescope (LLT) System, and (iii) the Laser Safety System. The Laser Service Enclosure (LSE), which houses the three $50 \mathrm{~W}$ lasers benches and the associated electronics, was mounted on the telescope elevation structure, requiring the lasers to work with a variable gravity vector. The BTO/LLT System was responsible for accepting the beams at the output of the laser system, transferring them along the telescope elevation structure (Optical Path) and then launching them from the Laser Launch Telescope located behind the TMT secondary mirror (LGSF Top End). The original design is presented in Figure 1.

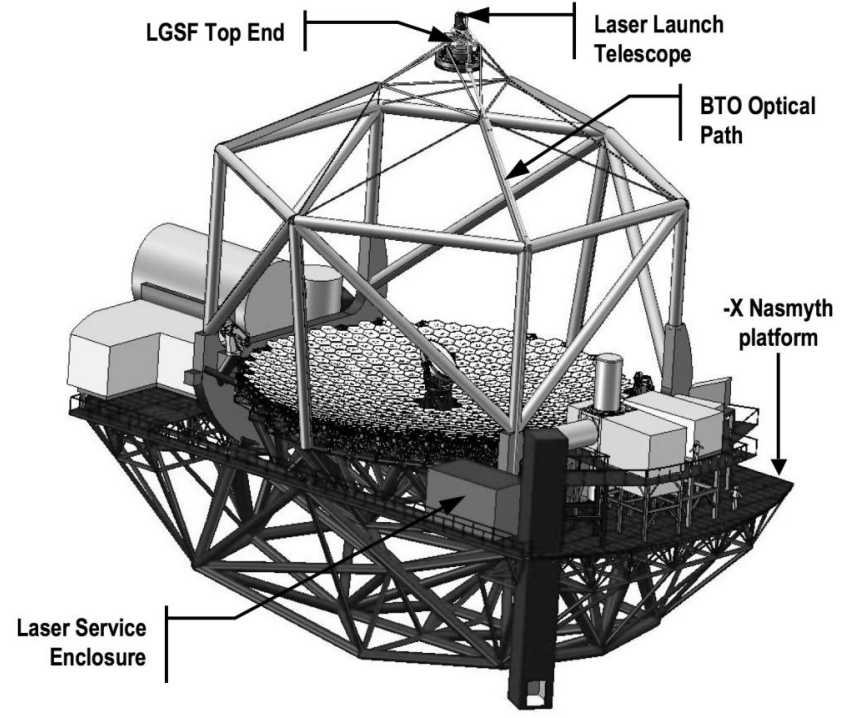

Figure 1: Original (2006) LGSF Conceptual Design

This paper presents the recent updates of the LGSF design. In particular, the LGSF Top End has been significantly redesigned to adapt for the new Ritchey-Chrétien optical design of the TMT, to compensate for the TMT telescope top end flexure, to optimize the number of optical surfaces and finally, to reduce the mass and volume. The LSE has been relocated within the telescope azimuth structure to allow the lasers to work in a fixed gravity orientation, to reduce both the wind obstruction on the telescope and the vibrations coupled into the telescope structure, and to facilitate the LSE access.

The conceptual design for the LGSF Top End is now sufficiently mature to permit us to define its Interface Control Documents to the other telescope sub-systems. However, the designs of the LSE and the BTO Optical Path are still 
described in terms of functional schematics and top-level layouts, and more work is needed to bring their designs to a comparable level of maturity before beginning the Preliminary Design phase of the LGSF project.

Section 2 of this paper presents the new optical layout that has been developed for the LGSF Top End and the BTO Optical Path. Section 3 focuses on the redesign of the LGSF Top End, while section 4 describes the trade study leading to the relocation of the Laser Service Enclosure within the telescope azimuth structure.

\section{LGSF OPTICAL LAYOUT}

The Laser Service Enclosure is now mounted into the telescope azimuth structure. The dimensions of the room have been modified to fit between the azimuth structure trusses as shown in Figure 2.

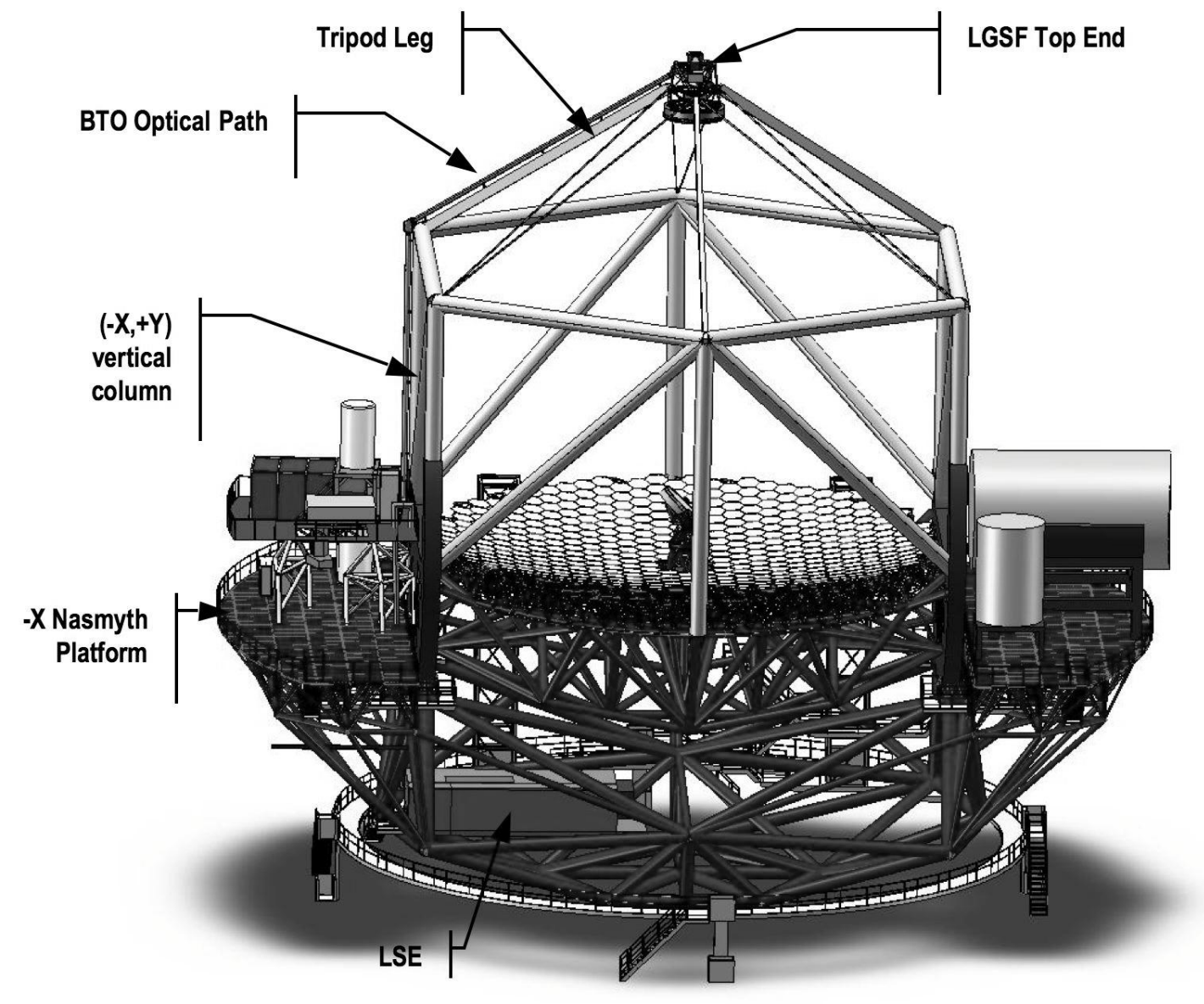

Figure 2: Updated LGSF Conceptual Design

At the output of each laser, the LSE Beam Splitter and Shutter Assembly splits the laser beam into either 2 or 3 beams, depending upon the asterism to be generated. The beams are formatted into a compact $3 \times 3$ configuration, and then directed up to the $-X$ Nasmyth Platform at a location beneath the telescope elevation axis via the LSE Fold Array.

From there, the beams are transferred across to the $-X$ elevation journal along the telescope elevation axis via two active steering mirror arrays (the Deployable Tracking Array and the Deployable Pointing Array) and a fold array (the Deployable Fold Array). The active arrays are used to follow the rotation of the telescope elevation structure as the zenith angle changes, direct the beams in the beamlines appropriate for each asterism, and to correct for any misalignments of the telescope top end due to thermal and flexure effects. The section of the optical path located along the elevation axis is flexible to allow the telescope to move in elevation, and is retractable to enable narrow-field, seeing- 
limited instruments to be mounted on the telescope elevation axis. This location on the Nasmyth platform would otherwise be vignetted by the beam duct.

Following the deployable path, the beams are directed along the elevation journal, reflected up along the $(-\mathrm{X},+\mathrm{Y})$ vertical column of the telescope elevation structure via the Truss Fold Array, and then up the connecting tripod leg via the Tripod Fold Array. Finally, the beam lines are folded by the Truss Centering Array to enter the LGSF Top End.

Three lenses located along the vertical column path are used to re-image the laser beams from the laser output port onto the entrance pupil of the Laser Launch Telescope.

The LGSF Top End, as illustrated in Figure 3, includes (i) the Diagnostic System, which monitors beam alignment with respect to the telescope top end as well as beam power, (ii) the asterism generator, which transforms the square $3 \times 3$ pattern of beams into the requested asterism, (iii) the $\mathrm{K}$ mirror, which acts as an image rotator and maintains a fixed asterism orientation in the LGS wavefront sensor focal plane, and (iv) the Laser Launch Telescope, which projects the beams to the sky.

The Laser Launch Telescope is installed on a pivot mount to allow first order correction of the pointing error due to telescope top end flexure.

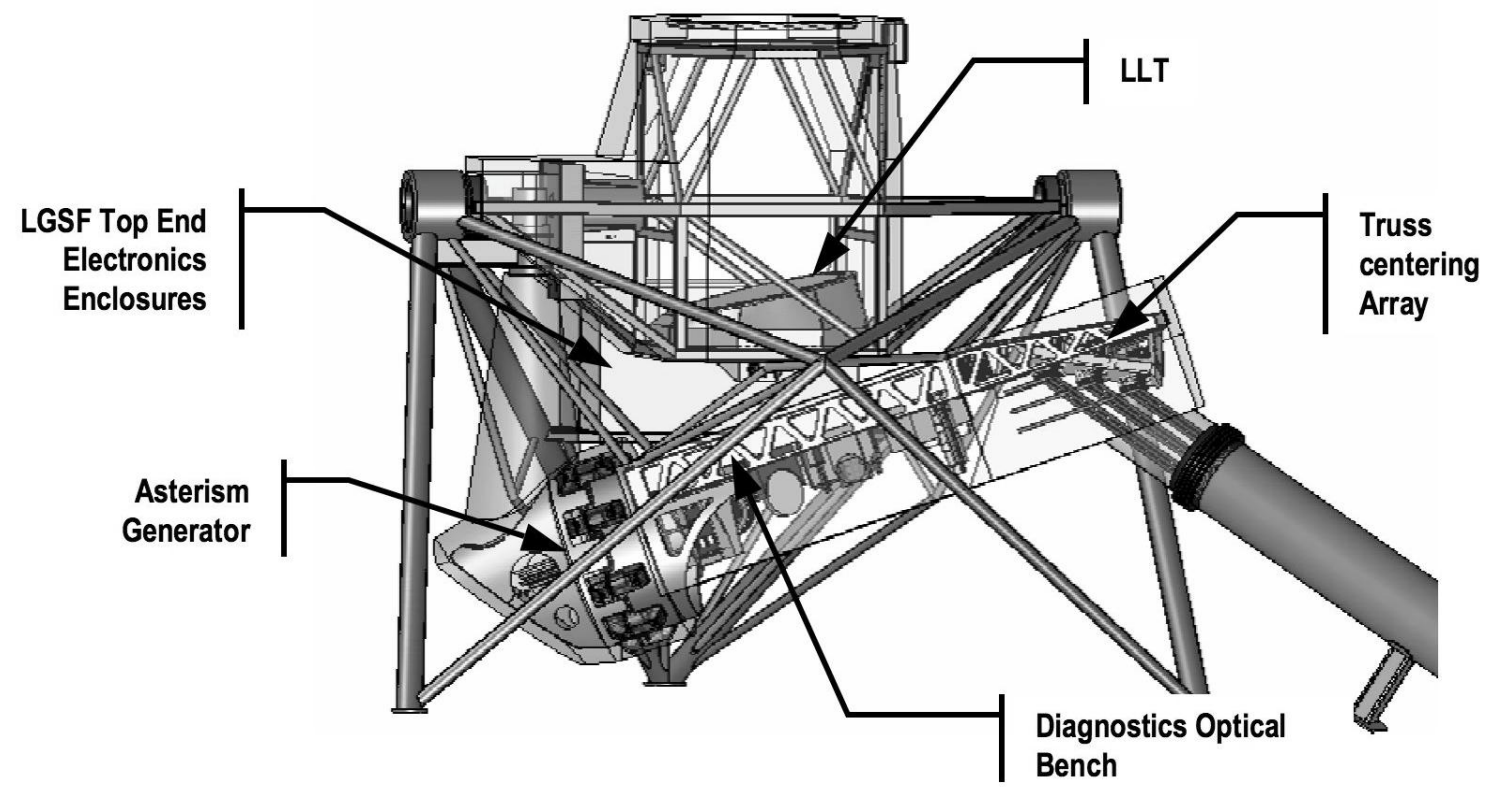

Figure 3: LGSF Top End in zenith pointing orientation as viewed from behind the telescope

An optical schematic of the LGSF is presented in Figure 4, including all of the optical sub-systems. For clarity, only three of the nine beams are shown after the LSE Fold Array. The cameras labeled "PAC" are used to verify coarse alignment of the laser beams before closing the flexure compensation loops using the diagnostics system measurements.

\footnotetext{
* Note that each beam line includes a separate shutter located at the output of the LSE to allow individual diagnostic power measurements.
} 


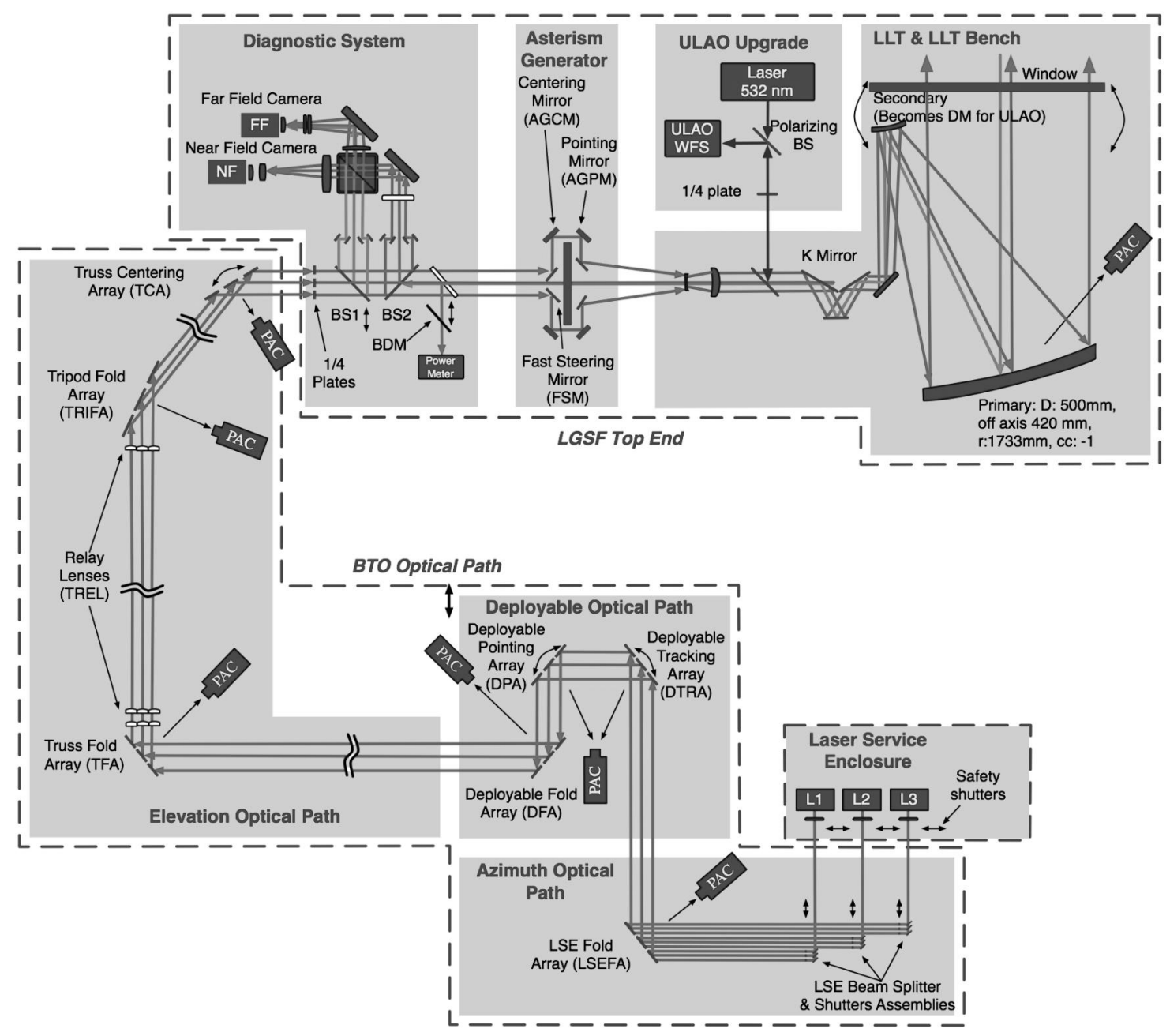

Figure 4: LGSF Optical Schematic

\section{LGSF TOP END REDESIGN}

\subsection{Flexure compensation system}

Since the LGSF Conceptual Design study, TMT has been updated to a Ritchey-Chrétien optical design and the secondary mirror support system has been defined. The telescope top end flexure has been analyzed and is now estimated to be on the order of $\pm 15 \mathrm{~mm}$ decenter and $\pm 2 \mathrm{mrad}$ tip/tilt in the TMT secondary coordinate system. The conceptual design of the LGSF Top End was revised, since neither the LLT field of view nor the dynamic range of the pointing and centering mirrors in the asterism generator was large enough to compensate for the estimated telescope top end flexure and maintain the asterism on axis with a good beam quality.

Note that the flexure of the telescope truss members due to variations in zenith angle is addressed via the Deployable Pointing Array and the Truss Centering Array as in the initial LGSF conceptual design. The dynamic range and clear aperture of these active mirrors will be defined in the next phase of the design.

The translation of the LLT with respect to the TMT optical axis can be neglected, since $15 \mathrm{~mm}$ at a range of $100 \mathrm{~km}$ corresponds to an angle of approximately $\sim 31$ mas. However, the $2 \mathrm{mrad}$ of tip/tilt corresponds to $412 \mathrm{arcsec}$, which is significantly larger than the LGS position accuracy requirement imposed by the LGS wavefront sensor acquisition field of view located within the AO systems. Several options were considered for the tip/tilt correction:

- Using the asterism generator centering and pointing mirrors of the LGSF Top End. This option was rejected, because of the large angular corrections needed. 
- $\quad$ Using the LLT M2/M3 mirrors. The LLT components are presented in Figure 5. In this option, the input flat M3 is tilted by an amount equal to the flexure tilt component, and M2 is tilted in the opposite sense to compensate for the LLT image degradation produced by tilting M2. This option results in a tilt of the input focal plane, which would require additional optical adjustments in the BTO. Consequently, this option was not investigated further.

- $\quad$ Rotating the LLT M1/M2 mirrors. This option consists of tilting the LLT M1 and counter-tilting the LLT M2 to correct for most of the image degradation. Both mirrors will rotate around the LLT Y-axis on their respective vertices since the TMT flexure will be in the LLT X-axis direction. The resulting image quality of the LLT is degraded but acceptable, however the mechanical implementation looks complicated and the option was rejected.

- Tilting the LLT assembly. This is the proposed solution; it is a simpler solution to implement, and it consists of tilting the entire LLT assembly by an amount necessary to compensate for the flexure tilt

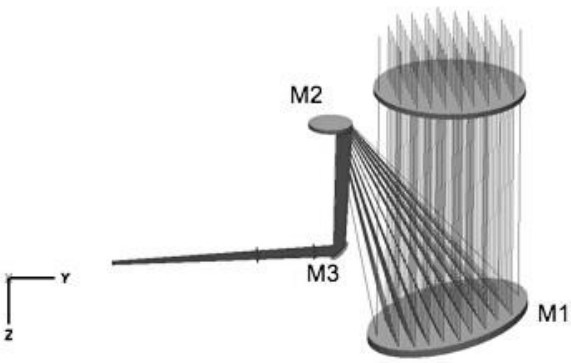

Figure 5: LLT components, showing the local coordinate system. The LLT is an off-axis reflective telescope with a system magnification of 60 . component. The LLT is installed into a rotating frame, allowing the LLT to remain in a fixed alignment and to be aligned separately if necessary. The space frame supporting the LLT ends into two flexures, which mount onto the LGSF Top End framework as illustrated in Figure 6. The axis of the rotation is coincident with the input optical axis, so the LLT can be tilted to compensate for the telescope top end flexure without any optical re-alignment. A linear actuator will be implemented and will apply a force to the bottom of the LLT frame perpendicular to the rotational axis of the flexure mounting. The requirements of the linear actuator have been defined and are summarized in Table 2. Potential candidates for the linear actuator have been identified.
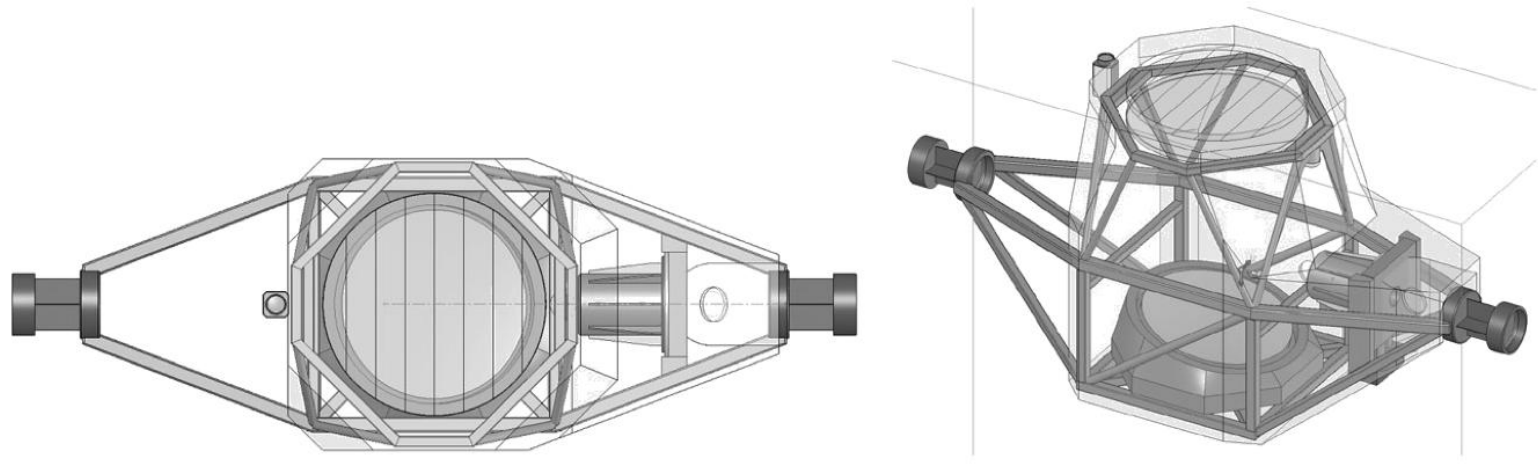

Figure 6: Top view (left) and isometric view (right) of the LLT mounted in its space frame, with two flexures along the input optical axis.

Table 2: LLT Flexure Compensation System Requirements

\begin{tabular}{|l|l|}
\hline LLT Rotation & $3.0 \mathrm{mrad}$ (conservative) \\
\hline Moment arm, actuator to tilt axis & $551 \mathrm{~mm}$ \\
\hline Minimum actuator travel & $1.65 \mathrm{~mm}$ \\
\hline Design actuator travel & $3.00 \mathrm{~mm}$ \\
\hline Linear resolution for 1 arcsec tilt & $2.7 \mu \mathrm{m}$ \\
\hline Repeatability & $5.0 \mu \mathrm{m}$ \\
\hline Minimum force & $\pm 100 \mathrm{~N}$ (or $0-200 \mathrm{~N}$ in one direction) \\
\hline Minimum axial stiffness & $3.85 \mathrm{~N} / \mu \mathrm{m}$ \\
\hline
\end{tabular}




\subsection{Mating the BTO elevation optical path to the LGSF top end}

One of the goals of the LGSF top end redesign was to simplify the task of directing the beams from the BTO optical path into the LGSF Top End using a minimum number of fold mirrors and without vignetting the TMT primary.

Because the BTO Optical Path travels up the $(-\mathrm{X},+\mathrm{Y})$ truss vertical column and then transfers to the connecting tripod leg, the beams intercepts the LGSF Top End at a $30^{\circ}$ angle from the negative $\mathrm{X}$-axis (looking down from the top when the telescope is pointed at zenith). To solve this issue, the diagnostic optical bench and asterism generator sub-assembly were rotated in the $\mathrm{XY}$ plane to accept the beam coming up the truss, and tilted to minimize the angle of incidence on the centering mirrors located at the top of the tripod leg.

Figure 7 illustrates how the BTO Optical Path (top left arrow) interfaces with the LGSF Top End. In this figure, the TMT X-axis runs horizontally through the LLT. The orientation of the LLT remains oriented along the X-axis, while the diagnostic optical bench and asterism generator are rotated by approximately $25^{\circ}$ about the vertical optical axis (right arrow) at the output of the asterism

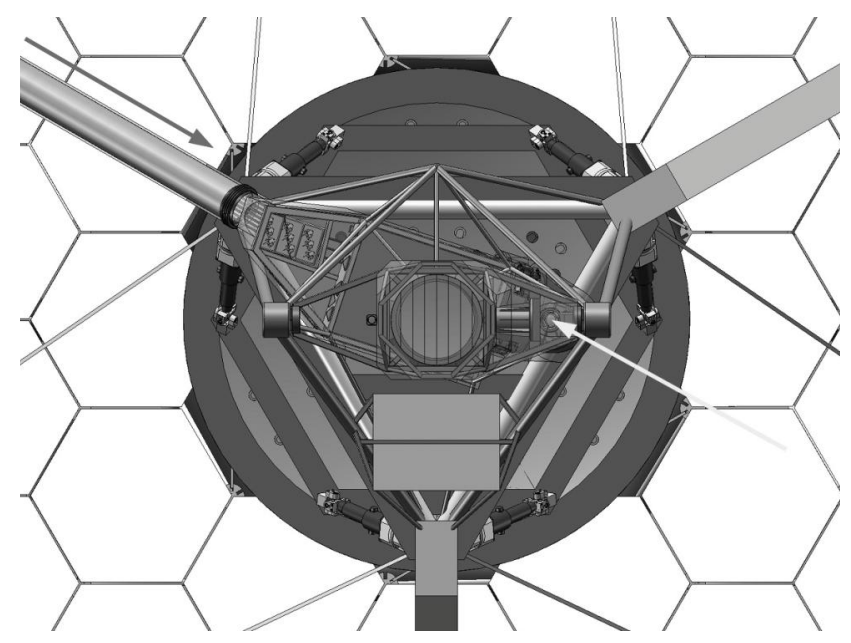

Figure 7: Top view of the LGSF Top End generator.

Figure 3 shows how the diagnostics optical bench and asterism generator have been tilted to reduce the angle of incidence on the truss centering array. The angle of incidence is now around $63^{\circ}$.

\subsection{Diagnostics system and asterism generator redesign}

The diagnostics system samples $0.5 \%$ of each laser beam through a beam splitter (BS2) into two cameras for near field and far field measurements. These cameras are used to measure the intensity profile and quality of each beam, and to provide the alignment error signals used to drive the truss pointing and centering arrays of the BTO Optical Path in closed loop. The Diagnostics System contains a second beam splitter (BS1) to direct the light of a natural star backward through the LLT into the two cameras to align the pointing of the LGSF with the telescope. An illustration of the diagnostics optical bench is given in Figure 8.

The nine laser beams are transported from the Laser Service Enclosure in a $3 \times 3$ square configuration (70mm centers, $140 \mathrm{~mm}$ side). This square pattern needs to be compacted into a smaller $3 \times 3$ square pattern $(25 \mathrm{~mm}$ centers) to make efficient use of the Near Field diagnostics camera CCD. The original LGSF design included two optical periscopes located before and after the diagnostic system in the high-power laser beam path. The first periscope reduced the size of the square pattern and the second periscope expanded it back to a larger pattern for input to the asterism generator. The new design maintains the large size square pattern $(70 \mathrm{~mm}$ centers) in the high-power laser beam path, and moves the first periscope within the diagnostics system after the beam splitter BS2. This removes 4 mirrors per beam in the high-power laser path, improving the overall LGSF throughput and reducing the number of surfaces with a high-power laser coating requirement. The beam splitters BS1 and BS2 are no longer single optical components but arrays of nine smaller beam splitters installed in a frame. In addition, BS1 can be moved into (during calibration) and out of (during operation) the high-power laser path with a simple two-position mechanism. If necessary, a second periscope could be implemented in the natural guide star path to allow verifying the alignment of the off axis beams with off axis natural guide stars.

Each beamline includes a quarter-wave plate to produce circularly polarized output beam that will maximize the photon return from the sodium layer. The quarter-wave plates are now located on the diagnostic optical bench just before BS1 as shown on Figure 8.

Finally, the beam dump assembly and power meter have been moved within the diagnostics optical bench, prior to the asterism generator. This was done in part for packaging reasons and in part for safety considerations, since the beam dump assembly now permits diagnostics of the high-power laser beams without propagating them into the asterism 
generator and the LLT collimator optics. The beam dump assembly consists of an array of small mirrors and holes installed in a frame. The beam dump assembly slides into the frame between the in and out position.

The asterism generator transforms the square $3 \times 3$ pattern into the requested asterism. It is composed of a series of four fixed and moving mirrors for each beam. For each beam, two of the mirrors are controllable in tip and tilt at low bandwidth to maintain the centering of the beams on the LLT pupil as well as the pointing of the LGS on the sky. The asterism generator also includes one fast steering mirror per beam, which is used to compensate LGS pointing jitter as measured by the associated LGS wavefront sensor in the AO system.

The asterism generator was scaled down by $15 \%$ to save weight, and to match its focal length to the space between it and the LLT collimator. The focal length of the LLT collimator was reduced proportionally to maintain the 60:1 magnification of the LLT optics. The large folding flat at the output of the asterism generator was replaced by an array of smaller mirrors and the entire asterism generator was slightly tilted to avoid interference between one of the larger AO asterism beam and a mirror sub-assembly. The revised design of the asterism generator and the output fold array is detailed in Figure 9.

The total weight of the LGSF Top End, including the truss centering array, the diagnostics optical bench, the asterism generator, the LLT assembly, the electronics assembly and the LGSF Top End support structure has been reduced to $\sim 900 \mathrm{~kg}$, which meets the LGSF Top End weight requirement of $1000 \mathrm{~kg}$.

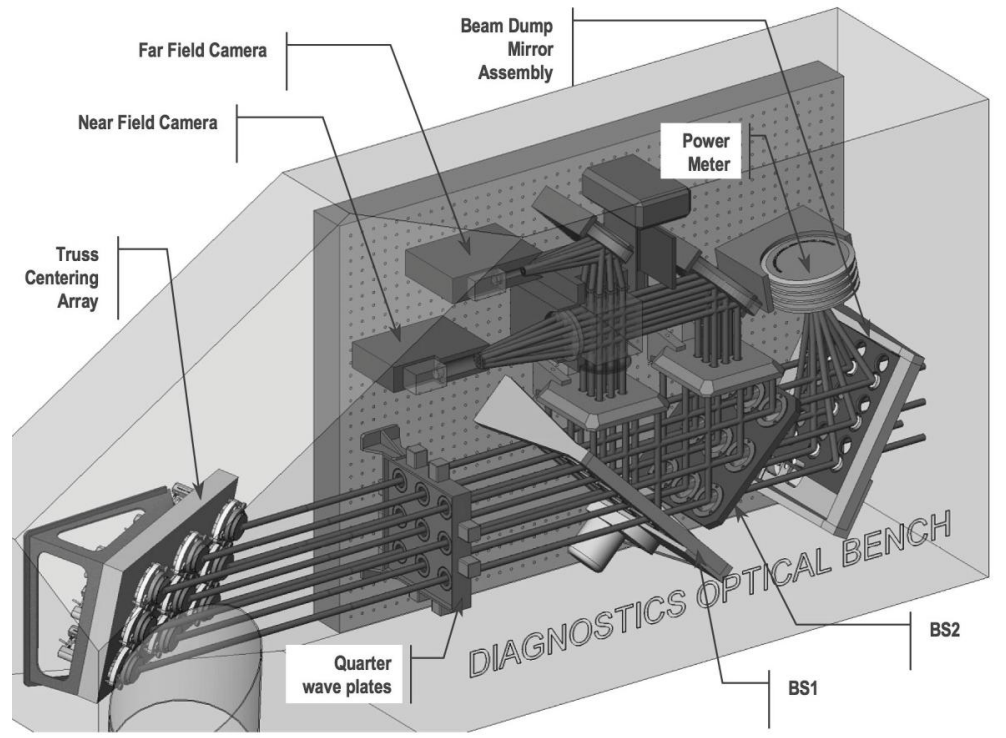

Figure 8: Diagnostics Optical Bench

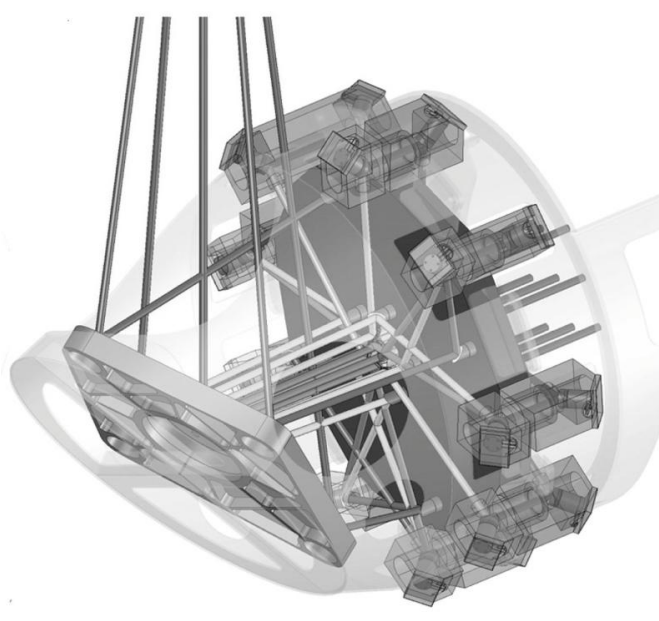

Figure 9: Asterism Generator

\subsection{LGSF Top End flexure analysis and modal analysis of seismic events}

An analysis of the LGSF and telescope flexure was performed to study the displacements and rotations of the LGSF elements due to gravity as the telescope moves in elevation. The approach used to compute the deflections assumes a linear behavior of the telescope and LGSF models:

- Calculate the deflections for the load case in which one turns gravity on and off with the telescope pointing at zenith,

- Calculate the deflections for the load case in which one turns gravity on and off with the telescope pointing at a zenith distance of 65 degrees,

- Linearly combine the two results by subtracting the zenith-pointing deflections from the 65 degrees-pointing deflections.

The global static tilts and displacements of the LGSF top end between 0 and 65 degrees are very similar to the telescope top end flexure compensation requirements, with approximately $2.2 \mathrm{mrad}$ of LLT axis rotation and $20 \mathrm{~mm}$ of displacement. The differential tilts and displacements within the top end are smaller by about an order of magnitude, and 
consist primarily of rigid body motion of the diagnostic optical bench with respect to the rest of the LGSF Top End. Optical tolerance analysis and raytracing will be needed to translate these misalignments into (i) the required adjustments to the pointing and centering arrays, (ii) the required sizes of the BTO optics, and (iii) the residual beam misalignments and aberrations which are not properly corrected by the pointing/centering arrays because the LGSF top end does not behave precisely as a rigid body. The last effect may be studied independently, and it may be reduced in magnitude by redesigning the diagnostics optical bench support structure if necessary.

In addition to the gravitational flexure, the lower modal frequencies of the LGSF structure were calculated to establish the sensitivity of the structure to earthquake loads based on the spectrum provided by TMT. The results are presented in Table 3.

Table 3: LGSF Top End modal analysis results

\begin{tabular}{|c|c|c|}
\hline Mode frequency & Description & Proposed corrective actions \\
\hline Mode $1(13.9 \mathrm{~Hz})$ & $\begin{array}{l}\text { This is a side-to-side motion of the truss member } \\
\text { supporting the electronics cabinets, amplified by the } \\
\text { weight }(256 \mathrm{~kg}) \text { of the electronics cabinets themselves. } \\
\text { This drives a twisting motion of the two flexures } \\
\text { supporting the LLT. }\end{array}$ & \multirow[t]{2}{*}{$\begin{array}{l}\text { Stiffening the electronics cabinet } \\
\text { support frame will increase th } \\
\text { frequencies of these two modes an } \\
\text { will reduce the excitation produced by } \\
\text { the wind loading spectrum. }\end{array}$} \\
\hline Mode $2(16.0 \mathrm{~Hz})$ & $\begin{array}{l}\text { This is a motion in the Y direction of truss members } \\
\text { supporting the electronics cabinets, also resulting from } \\
\text { the weight of the electronics cabinets. This drives a tilt } \\
\text { motion of the LLT structure in the same direction. }\end{array}$ & \\
\hline Mode $3(20.8 \mathrm{~Hz})$ & $\begin{array}{l}\text { This is a rocking motion of the diagnostics optical } \\
\text { bench and asterism generator sub-assembly, which also } \\
\text { drives a small motion of the LLT frame. }\end{array}$ & $\begin{array}{l}\text { Modification of the members } \\
\text { supporting this sub-assembly could } \\
\text { increase the frequency of this mode. }\end{array}$ \\
\hline
\end{tabular}

The accelerations of these 3 LGSF modes have been estimated: $6.6 \mathrm{~g}$ for the lowest mode and between 5 and $6 \mathrm{~g}$ for the two other modes. The corrective actions proposed in the $3^{\text {rd }}$ column of Table 3 are not likely to reduce significantly the local accelerations and it is expected that the local accelerations will still be in the range of 5 to $6 \mathrm{~g}$.

In addition to the proposed list of corrective actions, the next phase of the LGSF design should also study how to minimize potential damage of the LGSF Top End components, telescope sub-systems, and personnel during an earthquake.

\section{LASER SERVICE ENCLOSURE LOCATION}

The original conceptual design for the LGSF includes a Laser Service Enclosure (LSE) mounted on the $-X$ elevation journal of the telescope as described in Figure 1. The main advantage of this location is the very simple optical path needed to transport the laser beams to the telescope top end for projection to the sky. However, there are several downsides with this approach:

- The lasers must operate in a variable gravity vector,

- The LSE inhibits the airflow across the primary, particularly when the telescope is moving in elevation,

- The LSE vibrations couple into the telescope elevation journal and the M1 structure,

- Access to the room is limited to specific telescope orientation (zenith pointing).

Concerns about the prospects for acquiring $50 \mathrm{~W} \mathrm{CW}$ or mode locked $\mathrm{CW}$ lasers operable with a variable gravity vector motivated us to revisit the location of the LSE. Several options were considered for relocating the LSE:

- $\quad$-X Nasmyth platform location: the LSE is mounted on the -X Nasmyth platform instead of the -X telescope elevation journal, and in consequence does not move in elevation with the telescope. This solution requires a significantly more complex optical path with the laser beams first sent along the elevation journal toward the elevation axis, then across the elevation axis before being transported back along the $-\mathrm{X}$ elevation journal and 
then up the telescope elevation structure. Because a small portion of the new optical path follows the telescope elevation axis, this portion of the path (called Deployable Optical Path) needs to be retracted when the telescope is observing with an on-axis instrument. On the plus side, this solution allows the lasers to operate in a fixed gravity vector, reduces somewhat the wind obstruction across the primary mirror and the vibrations coupled into the telescope elevation structure, and provides unlimited access to the LSE.

- Azimuth structure location: the LSE is mounted within the (-X, $-\mathrm{Y})$ telescope azimuth structure as shown in Figure 2. This solution has several advantages: (i) the lasers are operated in a fixed gravity vector, (ii) the wind obstruction above M1 is eliminated, (iii) the vibrations coupled on the telescope structure are significantly damped and (iv) personnel access is not limited to a particular telescope orientation but requires new stairways and walkways. This option, like the previous option, requires a Deployable Optical Path, with a slightly different geometry. Access to the LSE for installation and maintenance of the lasers (or of the LSE itself) looks more complicated, however.

Other locations on the $-\mathrm{X}$ Nasmyth or $+\mathrm{X}$ Nasmyth platforms were rejected because they would use space reserved for science instrumentation. Location within the $+X$ telescope azimuth structure was rejected because the beam path would prevent the implementation of a Ground Layer Adaptive Optics (GLAO) LGS System for the TMT WFOS instrument ${ }^{3}$, which is currently located on the telescope elevation axis.

The azimuth structure location is the preferred option. As illustrated in Figure 2 and Figure 10, there is enough space to fit a rectangular laser room of dimension $12.3 \mathrm{~m}$ (W) $\times 4.8 \mathrm{~m}(\mathrm{D}) \times 2.9 \mathrm{~m}(\mathrm{H})$, with additional space at the margins of this volume for walkways and other equipment. As shown in Figure 10, there is also additional space available in the $(-\mathrm{X},+\mathrm{Y})$ telescope azimuth structure to add a somewhat smaller second LSE. A 2 T monorail crane will be used for the installation of the LSE itself and the installation/removal of the lasers and laser electronics to/from the LSE. The monorail crane will be located above the LSE and will be able to reach the observing floor.

The mass and volume of the LSE have been defined based upon the Lockheed Martin Coherent Technologies 50W laser, which is currently in fabrication for the Gemini

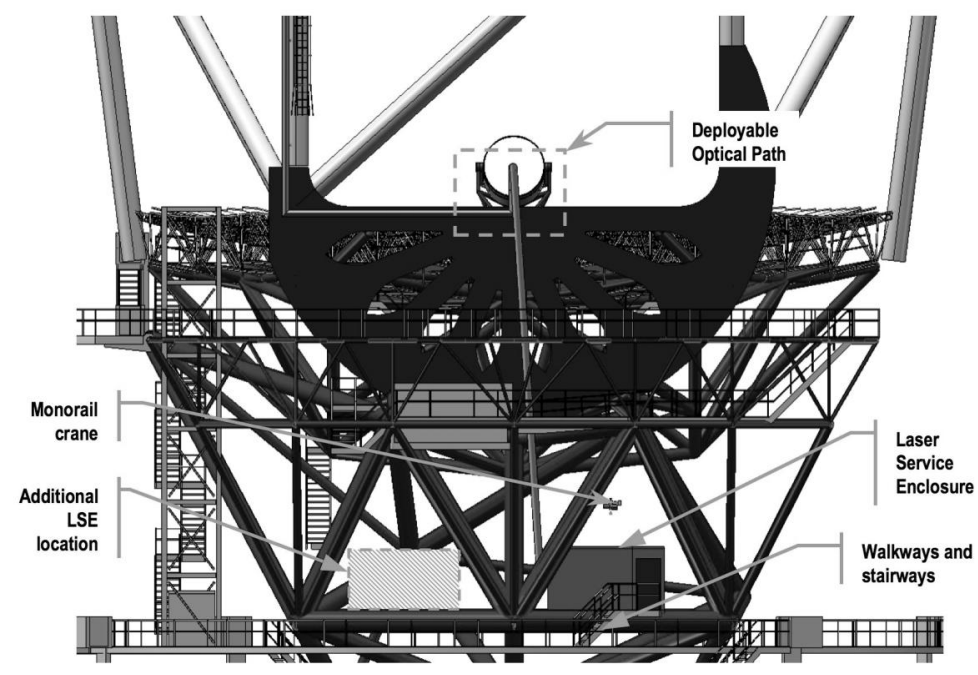

Figure 10: Location of the LSE within the telescope azimuth structure South telescope ${ }^{8}$.

The design of the optical path is still in progress. A few concepts have been defined and will be explored further:

- At the output of the LSE, the beam path is slightly tilted in the X and Y directions to directly intercept the elevation axis, in order to minimize the number of fold arrays needed within the path. However, interference with the instrument supports located on the $-\mathrm{X}$ Nasmyth platform will need to be resolved, as well as the attachment of the components to the telescope azimuth structure and access for cleaning and maintenance.

- The current layout of the deployable duct does not vignette the field of view of NFIRAOS, which is finally constrained by the elevation journal at zenith angles greater than 65 degrees.

- The Deployable Optical Path includes two active arrays and one fold array as described in Figure 4. Since the telescope elevation structure rotates, the laser beams must be steered as they cross from the Nasmyth platform onto the elevation structure. Each mirror in the Deployable Tracking Array located on the telescope azimuth structure side must be pointed to track its target mirror on the elevation structure. Each mirror in the Deployable Pointing Array located on the telescope elevation structure must then be pointed to align its laser beam along 
the remainder of the beam-path. Because this concept modifies the locations of the pointing and centering arrays in the original LGSF conceptual design, the relay lens optical design will also need to be revisited.

- The Deployable Optical Path is split into two sections that will be automatically retracted and folded when the LGSF is not in operation. The connection of these two sections will be implemented between the Deployable Tracking Array and the Deployable Pointing Array. It is envisioned at the moment that the azimuth portion of the Deployable Optical Path, including the Deployable Tracking Array, will be retracted down by at least 2 meters to a location entirely beneath the instrument servicing walkways, and the elevation portion of the Deployable Optical Path, which includes the Deployable Pointing Array, will be first rotated by 90 degrees and then folded down behind the elevation journal.

- The new LGSF design will utilize 143 actuators (106 servo motors, 19 DC motors and 18 piezoelectric actuators) and will monitor approximately 50 sensors. This represents a small $6 \%$ increase of the total number of actuators in comparison to the LGSF conceptual design. Even though a new array of active mirrors has been added, the functions of the laser switchyard component are now performed more efficiently via the LSE Beam Splitter and Shutter Assemblies and the Deployable Optical Path active arrays. The total number of sensors (temperature sensors, flow sensors, control switch sensors, etc.) has been increased by a fair amount, in proportion to the beam path length increase.

- The components of the BTO are spread across the telescope azimuth and elevation structures and require a highly-distributed control architecture, which will utilize commercially available motion controllers. The control of the BTO components has been split into 4 principal electronics assemblies (i) the LGSF Top End Electronics Assembly, (ii) two BTO Deployable Optical Path Electronics Assemblies (one for each section of the deployable optical path) and (iii) the LSE Electronics Assembly. Each of these assemblies consists of one or two electronics enclosures, several motion controllers and some power supplies. The BTO main controllers will be implemented in the LGSF Top End Electronics Assembly.

- The overall LGSF throughput is slightly improved (by 1.4\%), because the number of additional optical surfaces in the optical path is smaller than the number of deleted optical surfaces in the switchyard and in the LGSF Top End.

\section{CONCLUSION}

The LGSF Top End design has been significantly modified and has now reached the desired level of maturity needed to define interfaces with the other TMT sub-systems. A new location for the LSE within the telescope azimuth structure has been adopted to allow the operation of lasers with a fixed gravity vector. However, more work is needed to update the design of the BTO optical path (and in particular to develop further the deployable optical path concept), to define the interfaces between the optical path and the telescope structure, and to update the relay lens design. Additional telescope flexure analysis should be done including the BTO optical path, to validate the current design and to define the dynamic range and clear aperture requirements of each active and passive mirror array comprising the BTO system.

\section{ACKNOWLEDGEMENTS}

The authors gratefully acknowledge the support of the TMT partner institutions. They are the Association of Canadian Universities for Research in Astronomy (ACURA), the California Institute of Technology and the University of California. This work was supported as well by the Gordon and Betty Moore Foundation, the Canada Foundation for Innovation, the Ontario Ministry of Research and Innovation, the National Research Council of Canada, the Natural Sciences and Engineering Research Council of Canada, the British Columbia Knowledge Development Fund, the Association of Universities for Research in Astronomy (AURA) and the U.S. National Science Foundation.

\section{REFERENCES}

1 G. Sanders and J. Nelson, "The status of the Thirty Meter Telescope project", in Ground-based and Airborne Telescopes, L. Stepp and R. Gilmozzi ed., SPIE 7012, 2008 
2 B. Ellerbroek, S. Adkins, D. Andersen, J. Atwood, C. Boyer, P. Byrnes, R. Conan, L. Gilles, G. Herriot, P. Hickson, E. Hileman, R. Joyce, B. Leckie, M. Liang, T. Pfrommer, J.C. Sinquin, J.P. Veran, L. Wang, and P. Welle. "Progress towards developing the TMT adaptive optical systems and their components", in Adaptive Optics Systems, N. Hubin, C. Max and P. Wizinowich, ed, SPIE Proc 7015, 2008

3 D. Crampton, L. Simard, D. Silva, "Early Light TMT instrumentation" in Ground-based and Airborne Instrumentation for Astronomy II, I. Mclean and M. Casali ed., SPIE Proc 7014, 2008

4 G. Herriot, D. Andersen, J. Atwood, C. Boyer, P. Byrnes, B. Ellerbroek, L. Gilles, P. Hickson, B. Leckie, J.P. Veran, L. Wang, and P. Welle, "NFIRAOS - TMT's initial adaptive optics system", in Adaptive Optics Systems, N. Hubin, C. Max and P. Wizinowich, ed, SPIE Proc 7015, 2008

5 R. Joyce, C. Boyer, L. Daggert, B. Ellerbroek, E. Hileman, M.Hunten, M. Liang, "The Laser Guide Star Facility for the Thirty Meter Telescope", in Advances in Adaptive Optics II, B. L. Ellerbroek and D. B. Calia, ed., SPIE Proc $6272,1 \mathrm{H} 1(2006)$

6 C. d'Orgeville, F. Rigaut and B. Ellerbroek, "LGS AO Photon Return Simulations and Laser Requirements for the Gemini LGS AO Program”, in Adaptive Optics System Technology, SPIE Proc., 4007, 131 (2000)

7 C. d'Orgeville and al., "The Gemini South MCAO Laser Guide Star Facility: getting ready for first light", in Adaptive Optics Systems, N. Hubin, C. Max and P. Wizinowich, ed, SPIE Proc 7015, 2008

8 I. Lee, G. Moule, M. P. Jalali, N. Vanasse, K. W. Groff, N. Rogers, A. K. Hankla, J. Roush, C. d'Orgeville, S. M. Adkins, Z. Prezkuta, " $20 \mathrm{~W}$ and $50 \mathrm{~W}$ guidestar laser systems update for the Keck I and Gemini South telescopes", in Adaptive Optics Systems, N. Hubin, C. Max and P. Wizinowich, ed, SPIE Proc 7015, 2008 